often at long distances from the area in which the troops are based. These so-called "Fire Brigade" actions require the ability for rapid deployment of highly mobile troops, often over long distances. In addition, although the number of casualties are likely to be less because the number of troops deployed is reduced, the severity of the wounds now is greater due to the increased stopping power of modern weapons.

The advent of compact mobile computers has allowed sophisticated planning to be performed in the field. However, such equipment can break down, be lost, or destroyed, and so, it still is the best principle to keep planning as simple as possible. The requirement for rapid deployment has also increased the use of air transport. Air transport can be used to evacuate casualties. This will allow all but the most immediate surgery to be performed outside the battle zone, often in the soldier's own country. However, if large numbers of wounded are to be brought home, it is essential that adequate liaison is established with the civil medical authorities, so that the civilian population is not put at risk.

Rapid air transport also increases the availability of blood. Blood-transport boxes have been developed, allowing blood to be transported safely for periods in excess of 72 hours, and small, mobile thermoregulator blood banks are available, with capacities varying from 40 to 3,000 units of blood. Blood donation and processing now is carried out in the home blood transfusion centres, where the degree of quality control is much higher than would be possible under field conditions.

With supplies of blood being delivered from the home base, the need for field donor sessions is greatly reduced. However, bad weather or enemy action may prevent such deliveries and a fallback plan must be in place. Newly developed, solid-phase grouping and screening kits have improved both the convenience and safety of testing in the field. New additive solutions (OASs) are being developed that will extend the shelf life of concentrated red cells (RBCs) to longer than 3 months. These will reduce the demand for blood during the build-up phase of an operation, with a consequent reduction in wastage. The latest generation of cell salvage machines are so compact as to be easily deployed in a field role. Recent research has shown that cell salvage is a practical technique, even when the wound potentially is infected, as would occur in a missile injury to the abdomen. Cell-free, oxygen carrying resuscitation fluids (CFOCRFs) are about to be licensed for clinical use, one a haemoglobin solution and one perflourochemical. Both of these products can be stored at room temperature and have a shelf life in excess of two years.

Currently, it is impossible to transport platelets by air. However, with the increasing severity of blast injuries, disseminated intravascular coagulopathy (DIC) is encountered more frequently. Experience form the Gulf War showed that it is feasible to deploy the latest compact apheresis machines and produce platelets in the field. These machines also can provide fresh plasma, thus, obviating the need for freezers in which to store fresh frozen plasma. The US Army recently has developed a fibrin bandage, that can be used to pack wounds and arrests bleeding almost instantaneously. Once this becomes available commercially, it may have a dramatic effect on blood requirements and could reduce mortality from major trauma.

Keywords: blood; casualties; cell salvage; fibrin bandage; field; transport; war

Prehosp Disast Med 2001:16(3):S107-108.

\section{Plenary Session 3}

\section{The Use of Performance Indicators in Planning an Effective EMS System \\ A/Prof Jerry Overton, $M P A$}

Executive Director, Richmond Ambulance Authority

Associate Professor, Department of Emergency Medicine, Medical College of Virginia, Virginia Commonwealth University, USA

The planning, implementation, and management of Emergency Medical Services (EMS) systems historically have been influenced by local political, cultural, and economic conditions with minimal consideration given to the role of EMS as the initial provider of care in the health care continuum. Recently, evidence-based research has proven the potential of EMS for reducing mortality, and as a result, expectations have increased in the governmental and medical communities.

To meet expectations while maintaining economic viability, EMS systems continually must assess performance and outcomes. The development of performance indicators is the foundation for establishing strategies for planning and implementing an effective EMS delivery system. The identification of accurate data sources, establishment of predetermined measurement intervals, and the reporting of relevant information are prerequisite for establishing effective performance indicators.

Once developed, performance indicators assess current performance, identify existing and potential problems, and assist in the proactive development of solutions. Understanding performance indicators and their application can impact all phases of EMS administration. The measurement of basic and advanced clinical procedures, response methodologies, response time intervals, resource development and utilization, and cost per transport are specific indicators that provide necessary tools for system management. Results from studying these specific components, along with others, then can be reviewed collectively to help establish a strategy for the design and implementation of an optimum system design that meets the needs of the patient and community.

Keywords: emergency medical services; EMS; performance indicators; planning; outcome Prebosp Disast Med 2001:16(3):S108. 